\title{
Antithrombotic therapy in very elderly patients with atrial fibrillation: Is it enough to assess thromboembolic risk?
}

This article was published in the following Dove Press journal:

Clinical Interventions in Aging

18 May 2010

Number of times this article has been viewed

\author{
Yutao Guo ${ }^{*}$ \\ Qiang $\mathrm{Wu}^{2^{*}}$ \\ Lu Zhang' \\ Tingshu Yang' \\ Ping Zhu' \\ Wenqian Gao' \\ Yuexiang Zhao' \\ Meng Gao' \\ 'Department of Geriatric Cardiology, \\ ${ }^{2}$ Department of Clinical Administration, \\ General Hospital of The Chinese PLA, \\ Beijing, China; *Shared equal authorship
}

Correspondence: Yutao Guo Department of Geriatric Cardiology, General Hospital of The Chinese PLA, 28 Fu Xing Road, Hai Dian District, Beijing, China, 100853

Tel +86 I06 6876924

$\mathrm{Fax}+86108828$ 327।

Emaildor_guoyt@hotmail.com

\begin{abstract}
Although attention has been given to thromboprophylaxis for atrial fibrillation (AF) in present treatment guidelines, practical, clinical antithrombotic therapy is poorly developed for very elderly patients. We reviewed the records of 105 consecutive patients with AF of mean age 85 years, to determine how the greatest benefits from antithrombotic therapy could be obtained in this group. The mean CHADS2 score in these patients was $3.1 \pm 1.5$. Before antithrombotic therapy, $21.0 \%$ of the patients had diseases with a risk of hemorrhage, $26.7 \%$ had diseases with a risk of thrombosis, and $8.6 \%$ had diseases with a risk of both hemorrhage and thrombosis. Moreover, 89 patients $(84.8 \%)$ were receiving a single antiplatelet drug, 10 (9.5\%) used aspirin plus clopidogrel, and six (5.7\%) were taking an oral anticoagulant (OAC). Additionally, dual antiplatelet therapy was more commonly given to patients with permanent $\mathrm{AF}$ (paroxysmal and persistent versus permanent, $6.3 \%$ and $12.5 \%$ versus $30 \%$, respectively, Chi-square $=8.4, P=0.010$ ). The incidence of adverse events was $25.7 \%$, with thromboembolic events in $20.0 \%$ and hemorrhage in $5.7 \%$ of patients. There were no thromboembolic events in those patients taking OACs, but $33 \%$ of patients who took OACs had bleeding complications. It is difficult to choose appropriate antithrombotic strategies in very elderly patients. Both the thrombotic risk and the bleeding risk should be considered for helping such patients derive optimal benefit from thromboprophylaxis for AF.
\end{abstract}

Keywords: atrial fibrillation, elderly, antithrombotic therapy, hemorrhage

\section{Introduction}

Atrial fibrillation (AF) is the most common cardiac arrhythmia, and it increases in prevalence with age, ranging from $0.5 \%$ in people aged $50-59$ years to almost $9 \%$ in people aged $\geq 80$ years. Heart failure, stroke, and thromboembolic events are its severe complications. ${ }^{1-3}$ When strokes occur in association with AF, patients have substantial mortality, morbidity, disability rates, and longer hospital stays. ${ }^{4}$ Given the increasing prevalence of AF and its associated thromboembolic complications, the present treatment guidelines (such as the Guideline for Prevention of Stroke in Patients with Ischemic Stroke or Transient Ischemic Attack, American College of Cardiology [ACC], American Heart Association [AHA], 2006; Antithrombotic Therapy in Atrial Fibrillation, ACCP8th, 2008) have focused on the use of thromboprophylaxis for $\mathrm{AF}^{5,6}$ Antithrombotic medicines include anticoagulant and antiplatelet drugs. The overwhelming consensus of the research has proven that the oral anticoagulant (OAC) warfarin is more effective than aspirin in preventing strokes for patients with AF. The management of antithrombotic treatment in the very elderly with an indication for warfarin is challenging, due to the need to balance carefully the 
risk of thromboembolism with that of hemorrhage. Thus, what is the situation in the real world for practical, clinical antithrombotic therapy according to the guidelines for AF in elderly patients? How can adequate and safe anticoagulation be achieved in an elderly population?

Scant data are available on the safety and efficacy of antithrombotic strategies that are adopted in elderly patients according to the guidelines. The present study aimed to determine the possible factors influencing the success of antithrombotic therapy in very elderly patients with AF, and to explore how the greatest benefits from antithrombotic therapy could be obtained in such a population group.

\section{Methods}

\section{Study population}

The study population was retrospectively identified using an electronic clinical information systems database. All demographic and laboratory data were obtained from medical records. Eligible patients were aged $\geq 70$ years. The patients were divided into three groups, ie, a group aged 70-79, a group aged 80-89, and a group aged over 90 years. Patients who had a pre-existing diagnosis of permanent, persistent, or paroxysmal AF and patients who developed new onset of AF during their current admission were included in this analysis. AF was diagnosed and classified according to the guidelines of ACC, AHA, and European Society of Cardiology (ESC). ${ }^{7}$ Exclusion criteria included rheumatic valvular heart disease and prosthetic heart valves. A follow-up was performed to ascertain outcomes, ie, episodes of hemorrhage, thrombotic events, and mortality. Hemorrhage included major and minor bleeding. Major bleeding was defined as an intracranial hemorrhage, a decrease in blood hemoglobin level of more than $5.0 \mathrm{~g} / \mathrm{dL}$, the need for a transfusion of two or more units of blood, the need for corrective surgery, or any combination of these events. Minor bleeding was defined as a subcutaneous ecchymosis or hematoma, gastrointestinal bleeding, or bloody sputum. Thrombotic events included stroke, transient ischemic attack, atrial thrombosis, pulmonary thromboembolism (PT), deep vein thrombosis (DVT), and peripheral vascular thrombosis. The stroke risk of our patients was assessed by the CHADS2 score (assigning one point each for the presence of congestive heart failure, hypertension, age 75 years or older, and diabetes mellitus, and assigning two points for a history of stroke or transient ischemic attack). ${ }^{8}$

\section{Statistical analysis}

Continuous variables were evaluated for normal distribution by the Kolmogorov-Smirnov test. The normally distributed continuous variables are shown as means \pm standard deviations (SDs), and nonparametrically distributed variables are shown as medians (interquartile ranges). Discrete variables are presented as frequencies (percentages). Discrete variables were compared via the Chi-square test or (when the $2 \times 2$ table had $<$ five patients) the Fisher test. Comparisons of the groups for continuous variables were performed with ANOVA for independent samples that were parametrically distributed. All $P$ values were two-sided, and a $P$ value of $<0.05$ was considered to be statistically significant. Analyses were performed with SPSS 13.0 (SPSS, Inc., Chicago, IL).

\section{Results}

\section{Patient characteristics}

Between 2001 and 2008, we reviewed 105 patients $(92.4 \%$ men, mean age of $84.9 \pm 6.1$ years) with AF, of whom 79 (75.2\%) had paroxysmal AF, $16(15.2 \%)$ had persistent AF, and $10(9.5 \%)$ had permanent AF. The population was older, with diabetes mellitus in $43.8 \%$, hypertension in $65.7 \%$, heart failure or impaired cardiac function in $51.4 \%$, and previous coronary events in $86.7 \%$. The mean CHADS2 score was $3.1 \pm 1.5$. Demographic characteristics and risk factors for stroke are presented in Table 1.

\section{Comorbid diseases in elderly patients with AF}

A total of $22.9 \%$ of the elderly patients suffered from cancer. Twenty-two patients $(21.0 \%)$ had diseases with a risk of hemorrhage, while $28(26.7 \%)$ had diseases with a risk of thrombosis. Additionally, nine patients $(8.6 \%)$ had diseases with a risk of both hemorrhage and thrombosis. Compared with the young elderly patients, the very elderly patients (aged over 90 years) had a significantly increased prevalence of diseases with risks of both hemorrhage and thromboses (Table 2).

\section{Antithrombotic drug regimen}

The mean antithrombotic therapy duration was $15.2 \pm 6$ years (range 0.7-32 years). Although the target INR (international normalized ratio) was 2.0 (range 1.6-2.5; AHA/ASA, 2006), INR values between 1.5 and 1.8 were considered adequate for very elderly patients aged over 80 years who were taking OACs. For patients who developed intracerebral hemorrhage after ischemic stroke, all anticoagulants and antiplatelet drugs were discontinued during the acute period for two weeks after the hemorrhage, and OAC usage was resumed after three months. Furthermore, for patients with recurrent thromboembolic events 
Table I Baseline characteristics of the study population

\begin{tabular}{|c|c|c|c|c|}
\hline Characteristic & Total sample $(n=105)$ & $70-79$ years $(n=22)$ & $80-89$ years $(n=64)$ & $\geq 90$ years $(n=19)$ \\
\hline Mean age, years (SD) & $84.9(6.1)$ & $76.2(2.9)$ & $85.2(2.7)$ & $93.5(2.7)$ \\
\hline \multicolumn{5}{|l|}{ Sex } \\
\hline Male, n (\%) & $97(92.4)$ & $21(95.5)$ & $58(90.6)$ & 18 (94.7) \\
\hline Female, n (\%) & $8(7.6)$ & I (4.6) & $6(9.4)$ & I (5.3) \\
\hline \multicolumn{5}{|l|}{ AF type } \\
\hline Paroxysmal AF, n (\%) & $79(75.2)$ & $19(86.3)$ & $44(68.8)$ & $16(84.2)$ \\
\hline Persistent AF, n (\%) & $16(15.2)$ & $2(9.1)$ & $12(18.8)$ & $2(10.5)$ \\
\hline Permanent AF, n (\%) & $10(9.5)$ & I (4.6) & $8(12.5)$ & I (5.3) \\
\hline \multicolumn{5}{|l|}{ Past medical history } \\
\hline Hypertension, n (\%) & $69(65.7)$ & $14(63.6)$ & $42(65.6)$ & $14(2||)$. \\
\hline Diabetes, n (\%) & $46(43.8)$ & $8(36.4)$ & $30(46.9)$ & $9(47.4)$ \\
\hline $\mathrm{CHF}, \mathrm{n}(\%)$ & $54(5 \mid .4)$ & $7(31.8)$ & $34(53.1)$ & $13(68.4)$ \\
\hline CAD, n (\%) & 91 (86.7) & $16(72.7)$ & $60(93.8)$ & I5 (79.0) \\
\hline Stroke/TIA, n (\%) & $24(22.9)$ & $3(13.6)$ & $10(15.6)$ & II (57.9) \\
\hline \multicolumn{5}{|l|}{ CHADS2 risk scores } \\
\hline Mean CHADS2 scores (SD)* & $3.1(1.5)$ & $2.4(1.3)$ & $3.0(1.4)$ & $4.1(1.4)$ \\
\hline Low (score 0), n (\%) & $2(0.02)$ & $2(0.09)$ & $0(0)$ & $0(0)$ \\
\hline Intermediate (score I-2) n (\%) & $41(39.1)$ & $9(40.9)$ & $29(45.3 \%)$ & $3(27.3 \%)$ \\
\hline High risk (score 3-6) n (\%) & $54(5 \mathrm{I} .4)$ & II (50.0) & 35 (54.7\%) & $8(72.7 \%)$ \\
\hline
\end{tabular}

Notes: CHADS2 score = one point each for the presence of congestive heart failure, hypertension, age 75 years or older, and diabetes mellitus, two points for a history of stroke or transient ischemic attack. *ANOVA, $F=8.2, P<0.05$. The $P$ value represents the comparison of the group aged $70-79$ years, the group aged $80-89$ years, and the group aged over 90 years.

Abbreviations: $\mathrm{AF}$, atrial fibrillation; $\mathrm{CHF}$, chronic heart failure; $\mathrm{CAD}$, coronary heart disease; $\mathrm{SD}$, standard deviation.

(such as DVT, PE, and venous thrombosis associated with the placement of a central venous catheter) who required anticoagulation soon after a cerebral hemorrhage, intravenous heparin treatment was given for two weeks, and OAC with a dosage of $1.5 \mathrm{mg} /$ day was continued, with rigorous monitoring and maintenance of INR in the lower end of the therapeutic range.

Of the 105 patients, 99 patients $(94.3 \%)$ were receiving antiplatelet drugs, whereas six patients $(5.7 \%)$ were taking OAC warfarin (Table 3). Dual antiplatelet therapy (aspirin plus clopidogrel) was more commonly given to patients suffering with permanent AF (Table 4).

\section{Adverse events with antithrombotic therapy in elderly patients}

The incidence of adverse events in elderly patients with $\mathrm{AF}$ was high (25.7\%), with hemorrhage in 5.7\% and thromboembolic events in $20.0 \%$ (Table 5). Among the hemorrhage cases, one case was major bleeding (in patients taking OAC), and five cases were minor bleeding events (Table 6).

Table 2 Comorbid diseases in elderly patients with atrial fibrillation before antithrombotic therapy

\begin{tabular}{|c|c|c|c|c|c|c|}
\hline & Total sample $(n=83)$ & $70-79$ years $(n=22)$ & $80-89$ years $(n=64)$ & $\geq 90$ years $(n=19)$ & $\chi^{2}$ & $P$ \\
\hline Cancer* n (\%) & $24(22.9)$ & $3(13.6)$ & $16(25.0)$ & $5(26.3)$ & 75.1 & 0.000 \\
\hline $\begin{array}{l}\text { Diseases with risk of } \\
\text { hemorrhage* } \mathrm{n}(\%)\end{array}$ & $22(21.0)$ & $3(13.6)$ & $12(18.8)$ & $7(36.8)$ & 71.1 & 0.000 \\
\hline $\begin{array}{l}\text { Diseases with risk of } \\
\text { thromboembolism* } n(\%)\end{array}$ & $28(26.7)$ & $4(18.2)$ & II (I7.2) & $13(68.4)$ & 93.6 & 0.000 \\
\hline $\begin{array}{l}\text { Diseases with risk } \\
\text { of hemorrhage and } \\
\text { thromboembolism*n (\%) }\end{array}$ & $9(8.6)$ & $2(9.1)$ & $3(4.7)$ & $4(2 I . I)$ & 145 & 0.000 \\
\hline
\end{tabular}

Notes: Cancers included meningioma, pharynx nasalis squamous carcinoma, suprahyoid gland adenoma, pulmonary cancer, gastric interstitialoma, gastric carcinoma, carcinoma of gastric cardia, liver cancer, sigmoid colon cancer, suprarenal epithelioma, kidney papillocarcinoma, carcinoma of the bladder, prostatic carcinoma and chronic lymphocytic leukemia. Diseases with a risk of hemorrhage were brain hemorrhage, hemorrhagic gastritis, duodenal ulcer, renal anemia and thrombocytopenia; in contrast, diseases with a risk of thromboembolism included lacunar cerebral infarction, vertebrobasilar artery insufficiency, atrial thrombosis, deep venous thrombosis, and peripheral vascular thrombosis.

*Chi-square test, $P<0.05$. The $P$ value represents the comparison of the group aged $70-79$ years, the group aged $80-89$ years, and the group aged over 90 years. 
Table 3 Antithrombotic regimen adopted in elderly patients with atrial fibrillation

\begin{tabular}{|c|c|c|c|c|c|c|}
\hline & Total sample $(n=105)$ & $70-79$ years $(n=22)$ & $80-89$ years $(n=64)$ & $\geq 90$ years $(n=19)$ & $\chi^{2}$ & $P$ \\
\hline Aspirin*n (\%) & $42(40.0 \%)$ & $14(63.6 \%)$ & $20(31.3 \%)$ & $8(42.1 \%)$ & 33.5 & 0.000 \\
\hline Clopidogrel n (\%) & 47 (44.8\%) & $9(40.9 \%)$ & $33(51.6 \%)$ & $5(26.3 \%)$ & 10.2 & 0.003 \\
\hline Aspirin plus clopidogrel ${ }^{\dagger} \mathrm{n}(\%)$ & $10(9.5 \%)$ & I (4.6\%) & $9(14.1 \%)$ & 0 & 3.3 & 0.228 \\
\hline Warfarin $^{\dagger}$ n (\%) & $6(5.7 \%)$ & I (4.6\%) & $4(6.3 \%)$ & I (5.3\%) & 4.8 & 0.105 \\
\hline
\end{tabular}

Notes: *Chi-square test, $P<0.00$ I. The $P$ value represents the comparison of the group aged $70-79$ years, the group aged $80-89$ years, and the group aged $\geq 90$ years. 'Fisher's exact test.

\section{Discussion}

A population-based study in China revealed that $0.8 \%$ of Chinese are currently afflicted by AF, with an absolute number of over 10 million, and the prevalence of this disease will increase with the expansion of the elderly in this population. ${ }^{9} 10$ Based on the Chinese population census, the elderly ( $>60$ years) currently account for $11 \%$ of the general population, and the estimated number of AF patients in China is set to surpass 25 million. The present study illustrates that various antithrombotic drugs were used in very elderly Chinese patients with AF who had a mean age of 85 years. We showed that anticoagulants should have been used to prevent thromboembolic events in these elderly patients with $\mathrm{AF}$, as most of them were at high risk for stroke according to their CHADS2 scores (mean CHADS2 score of 3.1). ${ }^{11}$ In fact, most of the elderly patients in this study used aspirin or clopidogrel, while only $6 \%$ of patients used warfarin. This is in agreement with data from another retrospective study by Qi et al, involving 41 major hospitals and 9,297 AF patients in China, which showed that long-term treatment with anticoagulants was used in only $6.6 \%$ of patients. ${ }^{10}$ So a wide gap exists between the antithrombotic therapy guidelines for AF and actual clinical practice in the very elderly with AF in China.

Why is it difficult to choose the appropriate antithrombotic therapy regimen in very elderly patients? The main reason that antithrombotic therapy is difficult in elderly patients is that they tend to have more comorbidities. Before antithrombotic therapy began in this study, $21.0 \%$ of the patients had diseases with a risk of hemorrhage, $26.7 \%$ of the patients had diseases with a risk of thrombosis, and $8.6 \%$ patients had diseases with a risk of both hemorrhage and thrombosis. In addition, the risks of hemorrhage and thromboembolism are significantly increased in very elderly patients compared with young elderly patients. Those patients aged 90 years or more were five times more likely to develop diseases with risks of both hemorrhage and thromboembolism compared with those aged 80 years. For elderly patients who have hemorrhagic gastritis, duodenal ulcers, renal anemia, thrombocytopenia, or a past medical history of brain hemorrhage, it is quite difficult to choose appropriate antithrombotic therapy. Another reason for difficulty using antithrombotic therapy is that the incidence of cancer is high in very elderly patients $(22.9 \%$ in patients in this study). All of the above-mentioned factors, including the greater number of comorbidities, the higher risks of hemorrhage and thromboembolism, and shorter life expectancy, can influence decision-making regarding an antithrombotic strategy in very old patients.

In addition, our study illustrated that the incidence of adverse events after antithrombotic therapy in elderly patients with AF was high, with thromboembolic events in $21 \%$ and hemorrhage in $6 \%$. These results suggested that antiplatelet drugs for the very elderly with AF did not efficiently prevent thromboembolic events, and dual antiplatelet therapy also did not show any better thromboprophylactic effects compared with OAC. Our results are consistent with those of the ACTIVE-W trial. ${ }^{12}$ The survey revealed that the stroke rates for those patients even with a CHADS2 score $=1$ were $1.25 \%$ per year on aspirin and clopidogrel, and $0.43 \%$ per year on an anticoagulant, which represents nearly a three-fold difference. In a similar series of patients in the Birmingham Atrial Fibrillation Treatment of the Aged study, a substantial benefit of

Table 4 AF type and outcomes of antithrombotic therapy

\begin{tabular}{|c|c|c|c|c|c|}
\hline & Paroxysmal AF $(n=79)$ & Persistent AF $(n=16)$ & Permanent AF $(n=10)$ & $\chi^{2}$ & $\boldsymbol{P}$ \\
\hline Aspirin ${ }^{\dagger}$ (\%) & $35(44.3)$ & $6(37.5)$ & $I(I 0.0)$ & 6.4 & 0.041 \\
\hline Clopidogrel* n (\%) & $36(45.6)$ & $6(37.5)$ & $5(50.0)$ & 10.2 & 0.006 \\
\hline Aspirin plus clopidogrel ${ }^{\dagger} \mathrm{n}(\%)$ & $5(6.3)$ & $2(12.5)$ & $3(30.0)$ & 8.4 & 0.010 \\
\hline Warfarin ${ }^{\dagger}$ n (\%) & $3(3.8)$ & $2(12.5)$ & I (I0.0) & 5.7 & 0.064 \\
\hline
\end{tabular}

Notes: *Chi-square test. 'Fisher's exact test. The $P$ value represents the comparison of paroxysmal AF, persistent AF and permanent AF. Abbreviation: $A F$, atrial fibrillation. 
Table 5 Adverse events after thromboembolic therapy in elderly patients with atrial fibrillation

\begin{tabular}{lllll}
\hline & Total sample $(\mathrm{n}=\mathbf{2 9})$ & $\mathbf{7 0 - 7 9}$ years $(\mathrm{n}=\mathbf{2 2})$ & $\mathbf{8 0 - 8 9}$ years $(\mathrm{n}=\mathbf{6 4})$ & $\geq \mathbf{9 0}$ years $(\mathrm{n}=1 \mathbf{9})$ \\
\hline Hemorrhage, $\mathrm{n}(\%)$ & $6(5.7)$ & $3(13.6)$ & $3(4.7)$ & $0(0)$ \\
Embolism, $\mathrm{n}(\%)$ & $21(20.0)$ & $4(18.2)$ & $15(23.4)$ & $2(10.5)$ \\
\hline
\end{tabular}

an anticoagulant over aspirin was seen among elderly subjects, including those patients with CHADS2 scores of $1-2$ or $>2 .^{13}$ It is well known that thrombogenesis in AF is mainly coagulation factor-related, with red (fibrin-rich) clots predominating in AF, as illustrated in some studies in which anticoagulants significantly reduced thrombogenesis, whereas aspirin and clopidogrel did not. ${ }^{14,15}$ However, our study showed that $33 \%$ of patients taking OAC had bleeding complications, and falls and dementia were the common factors leading to intracranial hemorrhage in the elderly patients taking OAC. ${ }^{16,17}$

In clinical practice, there is still an indication for long-term OAC use for patients who have major bleeding events after antithrombotic therapy, but the "when" and "how" of resuming antithrombotic treatment are the challenging problems. Unfortunately, the optimal antithrombotic treatment for this population is currently unknown. There are no randomized studies assessing the best regimen in this setting. Generally speaking, for patients who develop intracerebral hemorrhage after ischemic stroke, OAC should be resumed after three to six months. For patients with recurrent thromboembolic events (such as DVT, PE, and venous thrombosis associated with the placement of a central venous catheter) who required anticoagulation soon after a cerebral hemorrhage, intravenous heparin treatment should be given for two weeks, followed by continued $\mathrm{OAC}$ with rigorous monitoring and maintenance of the INR in the lower end of the therapeutic range (AHA/ASA, 2006). However, for the patient in this study who had a new onset of peripheral vascular thrombosis at 40 days after intracerebral hemorrhage secondary to ischemic stroke, OAC treatment with a daily dosage of $1.5 \mathrm{mg}$ proved to be effective for prevention of thromboembolism, with rigorous maintenance of INR at no more than 1.8 .

Our results suggest the optimal antithrombotic therapy selection for this population must be mainly accomplished based on individual and accurate risk stratification for both thromboembolism and hemorrhage during therapy, not based only on the risks before treatment. Age over 75 years, hemorrhoids, rhinorrhagia, hemorrhagic gastritis, duodenal ulcers, renal anemia, thrombocytopenia, a hemorrhagic history, and INR intensity should be considered when assessing the patient's bleeding risk.

Additionally, the anticoagulation therapy must be very closely controlled in all patients who receive OAC, especially if a high bleeding risk is present. In our experience, maintaining the INR value within the range of 1.5-1.8 by frequent monitoring of OAC therapy patients was safe and effective in the very elderly. According to clinical practical guidelines for AF based on the consensus opinion of Chinese experts, the target INR should be sustained at 1.8 (range 1.5-2.0) in patients aged over 75 years who are at high risk of bleeding. However, in this study, maintaining INR between 1.6 and 1.8 was sufficient for preventing hemorrhage and thromboembolism in elderly patients who required long-term anticoagulation soon after bleeding events. The maximal tolerated dosage of OAC is likely to be lower for Chinese patients compared with Caucasian patients. A recent study by Shen et al showed that Asians with AF were at greater risk for warfarin-related intracranial hemorrhage. ${ }^{18}$

Moreover, for AF patients at high risk of arterial and venous thrombosis or multiple episodes of thrombosis (for

Table 6 Clinical outcomes in geriatric patients with atrial fibrillation receiving different antithrombotic therapy regimens

\begin{tabular}{|c|c|c|c|c|}
\hline Outcome & Aspirin $(n=43)$ & Clopidogrel $(n=47)$ & Aspirin + Clopidogrel $(n=10)$ & Warfarin $(n=6)$ \\
\hline \multicolumn{5}{|l|}{ Hemorrhage (\%) } \\
\hline Major bleeding, n (\%) & $0(0)$ & $0(0)$ & $0(0)$ & I (I6.7) \\
\hline Minor bleeding, n (\%) & $2(4.7)$ & $I(2.1)$ & I $(10.0)$ & I (I6.7) \\
\hline \multicolumn{5}{|l|}{ Thromboembolic events (\%) } \\
\hline Stroke/TIA, n (\%) & $5(11.6)$ & $6(12.8)$ & $2(20.0)$ & $0(0)$ \\
\hline Systemic embolus n (\%) & $3(7.0)$ & $4(8.5)$ & $\mathrm{I}(10.0)$ & $0(0)$ \\
\hline
\end{tabular}

Notes: Major bleeding was defined as an intracranial hemorrhage, a decrease in blood hemoglobin level of more than $5.0 \mathrm{~g} / \mathrm{dL}$, need for a transfusion of two or more units of blood, need for corrective surgery or any combination of these events. Minor bleeding was defined as a subcutaneous ecchymosis or hematoma, gastrointestinal bleeding or bloody sputum. Systemic emboli included atrial thrombosis, pulmonary thromboembolism, deep vein thrombosis, and peripheral vascular disease. Abbreviation: TIA, transient ischemic attack. 
example, patients with a history of chronic obstructive pulmonary disease, mechanical ventilation, varicosity, dehydration, aggravated heart failure, concurrence of DVT, or ischemic stroke), heparin treatment could be initiated for two weeks and followed with OAC.

Finally, further studies are required to find a more powerful tool for predicting the bleeding risk in everyday practice in this setting in order to achieve optimal antithrombotic therapy. Large, randomized studies are needed to explore the best therapeutic strategies in patients who have major bleeding events after antithrombotic therapy and who require antithrombotic treatment soon thereafter.

The limitations of this study were the small sample size and the possibility that the rates of events may have been underreported. Therefore, further large-sample studies are required to verify the recommendations made in terms of individualization of antithrombotic therapy for patients with a high risk of hemorrhage and thromboembolism.

\section{Acknowledgment}

This work was supported by grants from Beijing Natural Science Foundation (7093134) and the Special Foundation for Central Medical and Health Care in China (205). We thank the participants and all involved staff. We also thank Professor Chunyan Zhou, School of Basic Medical Sciences, Peking University Health Science Center, for her kind help in the preparation of this manuscript.

\section{Disclosure}

The sponsors did not have a role in the design or conduct of this study, the analysis of data, or preparation of the manuscript. The authors have no conflicts of interest to report.

\section{References}

1. Crandall MA, Bradley DJ, Packer DL, Asirvatham SJ. Contemporary management of atrial fibrillation: Update on anticoagulation and invasive management strategies. Mayo Clin Proc. 2009;84:643-662.

2. Spence JD. Stroke: Atrial fibrillation, stroke prevention therapy and aging. Nat Rev Cardiol. 2009;6:448-450.

3. Narumiya T, Sakamaki T, Sato Y, Kanmatsuse K. Relationship between left atrial appendage function and left atrial thrombus in patients with nonvalvular chronic atrial fibrillation and atrial flutter. Circulation. $2003 ; 67: 68-72$.
4. Go AS, Hylek EM, Phillips KA, et al. Prevalence of diagnosed atrial fibrillation in adults: National implications for rhythm management and stroke prevention: The AnTicoagulation and Risk Factors in Atrial Fibrillation (ATRIA) Study. JAMA. 2001;285:2370-2375.

5. Ralph LS, Robert A, Greg A, et al. Guidelines for prevention of stroke in patients with ischemic stroke or transient ischemic attack: A statement for healthcare professionals from the American Heart Association/ American Stroke Association Council on Stroke: Co-sponsored by the Council on Cardiovascular Radiology and Intervention: The American Academy of Neurology affirms the value of this guideline. Stroke. 2006;37:577-617.

6. Daniel ES, Gregory WA, James ED, et al. Antithrombotic Therapy in Atrial Fibrillation: American College of Chest Physicians EvidenceBased Clinical Practice Guidelines. 8th ed. Chest. 2008;133:546-592.

7. Lévy S, Camm AJ, Saksena S, et al. International consensus on nomenclature and classification of atrial fibrillation. Europace. 2003;5:119-122.

8. Gage BF, Waterman AD, Shannon W, Boechler M, Rich MW, Radford MJ.Validation of clinical classification schemes for predicting stroke. Results form the National Registry of Atrial Fibrillation. JAMA. 2001;285:2864-2870.

9. Zhou ZQ, Hu DY, Chen J, Zhang RH, Li KB, Zhao XL. An epidemiological survey of atrial fibrillation in China. Chin J Int Med (Chinese). 2004;26:491-494. Chinese.

10. Qi Wenhang. Retrospective investigation of hospitalized patients with atrial fibrillation in mainland China. Int J Cardiol. 2005;105:283-287.

11. Gage BF, Van WC, Pearce L, et al. Selecting patients with atrial fibrillation for anticoagulation stroke risk stratification in patients taking aspirin. Circulation. 2004;110:2287-2292.

12. Healey J, Hart RG, Pague P, et al. Risks and benefits of oral anticoagulation compared with clopidogrel plus aspirin in patients with atrial fibrillation according to stroke risk: The Atrial Fibrillation Clopidogrel Trial with Irbesartan for Prevention of Vascular Events (ACTIVE-W). Stroke. 2008;39:1482-1486.

13. Mant J, Hobbs R, Fletcher K, et al. Warfarin versus aspirin for stroke prevention in an elderly community population with atrial fibrillation (the Birmingham Atrial Fibrillation Treatment of the Aged Study, BAFTA): A randomised controlled trial. Lancet. 2007;370:493-503.

14. Choudhury A, Lip GY. Atrial fibrillation and the hypercoagulable state: From basic science to clinical practice. Pathophysiol Haemost Thromb. 2003-2004;33:282-289.

15. Kamath S, Blann AD, Chin BS, Lip GY. A prospective randomized trial of aspirin-clopidogrel combination therapy and dose-adjusted warfarin on indices of thrombogenesis and platelet activation in atrial fibrillation. J Am Coll Cardiol. 2002;40:484-490.

16. Jacobs LG, Billett HH, Freeman K, Dinglas C, Jumaquio L. Anticoagulation for stroke prevention in elderly patients with atrial fibrillation, including those with falls and/or early-stage dementia: A single-center, retrospective, observational study. Am J Geriatr Pharmacother. 2009;7(3):159-166.

17. Robert-Ebadi H, Le GG, Righini M. Use of anticoagulants in elderly patients: Practical recommendations. Clin Interv Aging. 2009;4:165-177.

18. Shen AY, Yao JF, Brar SS, et al. Racial/ethnic differences in the risk of intracranial hemorrhage among patients with atrial fibrillation. JAm Coll Cardiol. 2007;50:309-315.
Clinical Interventions in Aging

\section{Publish your work in this journal}

Clinical Interventions in Aging is an international, peer-reviewed journal focusing on evidence-based reports on the value or lack thereof of treatments intended to prevent or delay the onset of maladaptive correlates of aging in human beings. This journal is indexed on PubMed Central, MedLine, the American Chemical Society's 'Chemical Abstracts Ser-

\section{Dovepress}

vice' (CAS), Scopus and the Elsevier Bibliographic databases. The manuscript management system is completely online and includes a very quick and fair peer-review system, which is all easy to use. Visit $\mathrm{http}: / /$ www.dovepress.com/testimonials.php to read real quotes from published authors. 